\title{
ESTADO DA ARTE SOBRE O USO DE BIG DATA NO PDP SOB A PERSPECTIVA DO DATA-DRIVEN DESIGN VOLTADO À INOVAÇÃO
}

Valkiria Pedri Fialkowski (val.pedri@gmail.com) - Universidade Federal do Paraná (UFPR).

Thais Scaglione (thais.scaglione@hotmail.com) - Universidade Federal do Paraná (UFPR). Aguinaldo dos Santos (ㅁasantos@ufpr.br) - Universidade Federal do Paraná (UFPR).

\section{RESUMO}

Gerar produtos inovadores, de maneira sustentável, que atendam às expectativas de mercado, é um desafio. Neste contexto, o Big Data tem ganhado destaque ao possibilitar a rápida obtenção de informações acerca do comportamento do usuário que podem ser utilizadas para o gerenciamento do PDP (Processo de Desenvolvimento de Produto), apoiando a eficácia nas tomadas de decisões estratégicas. Assim, o objetivo deste artigo é apresentar uma análise crítica, exploratória e qualitativa da literatura, sobre estudos de Big Data para as macrofases do PDP através do DDD (Data-Driven Design) visando a inovação. Por meio do método de Revisão Bibliográfica, foi possível identificar lacunas e ênfases acerca dos temas abordados. Em particular foram analisadas as fontes, tipos e processamento dos grandes dados dentro das atividades nas macrofases do PDP. Os resultados apontam oportunidades de pesquisa para o DDD no PDP e de contribuições do uso do Big Data. No entanto, também demonstram a necessidade de novas linguagens e capacitações dentre os profissionais envolvidos e reestruturação do PDP para integrar de maneira sistemática e estratégica o uso de dados no seu processo.

Palavras chave: design de produto; big data; PDP; estado da arte; tecnologias da informação e comunicação. 


\section{INTRODUÇÃO}

O PDP é um grupo de atividades que tem por objetivo identificar oportunidades de mercado e atender essas expectativas (ROZENFELD et al., 2006). Alão (2020) destaca que a frequente mudança de comportamento dos usuários e aumento das conexões e da interdependência dos stakeholders envolvidos, reforça a complexidade e relevância de se encontrar estratégias que visem acompanhar esse processo. Assim, coletar e interpretar informações, rapidamente e com assertividade, sobre os usuários, tornou-se um desafio.

Neste caminho, os grandes dados digitais - Big Data - têm ganhado destaque, visto que ao empregar-se técnicas e métodos para analisá-los pode-se extrair informações relevantes (SPIEGEL, 2016). Esses dados são gerados por diferentes atores e dispositivos (gadgets), inclusive pelos próprios processos de manufatura e de experiência do usuário (SPIEGEL, 2016). Ademais, a utilização da informação por meio da análise digital pode ajudar a organização a mudar a cultura de tomada de decisão, aumentando sua eficácia (FRISK; BANNISTER, 2017) e oportunidades de inovação.

No contexto do Design, a aplicação de Big Data para gerar soluções é referida como DataDriven Design (DDD) (BERTONI, 2018). Entretanto, poucas aplicações do DDD são apresentadas na literatura e há uma discussão limitada sobre o papel de tais modelos no processo de Design visando a inovação (BERTONI, 2020).

Assim, o presente artigo buscou compreender o estado da arte acerca da utilização do Big Data no Design, levando à seguinte pergunta: Quais são as ênfases e lacunas na literatura qualificada sobre a aplicação de Big Data no PDP, e em particular para o DDD, visando a inovação?

\section{REVISÃO TEÓRICA}

O Big Data pode ser definido por cinco características, as 5Vs: volume, velocidade, variedade, veracidade e valor (DEVI, 2019). No âmbito do Design, alguns termos emergentes da Tecnologia da Informação e Comunicação (TIC) que vêm sendo utilizados com mais frequência são: Data Science, Data Mining e Business Intelligence (PRIOR, 2019). Algumas das principais interações entre esses termos são elucidadas na figura 1. 


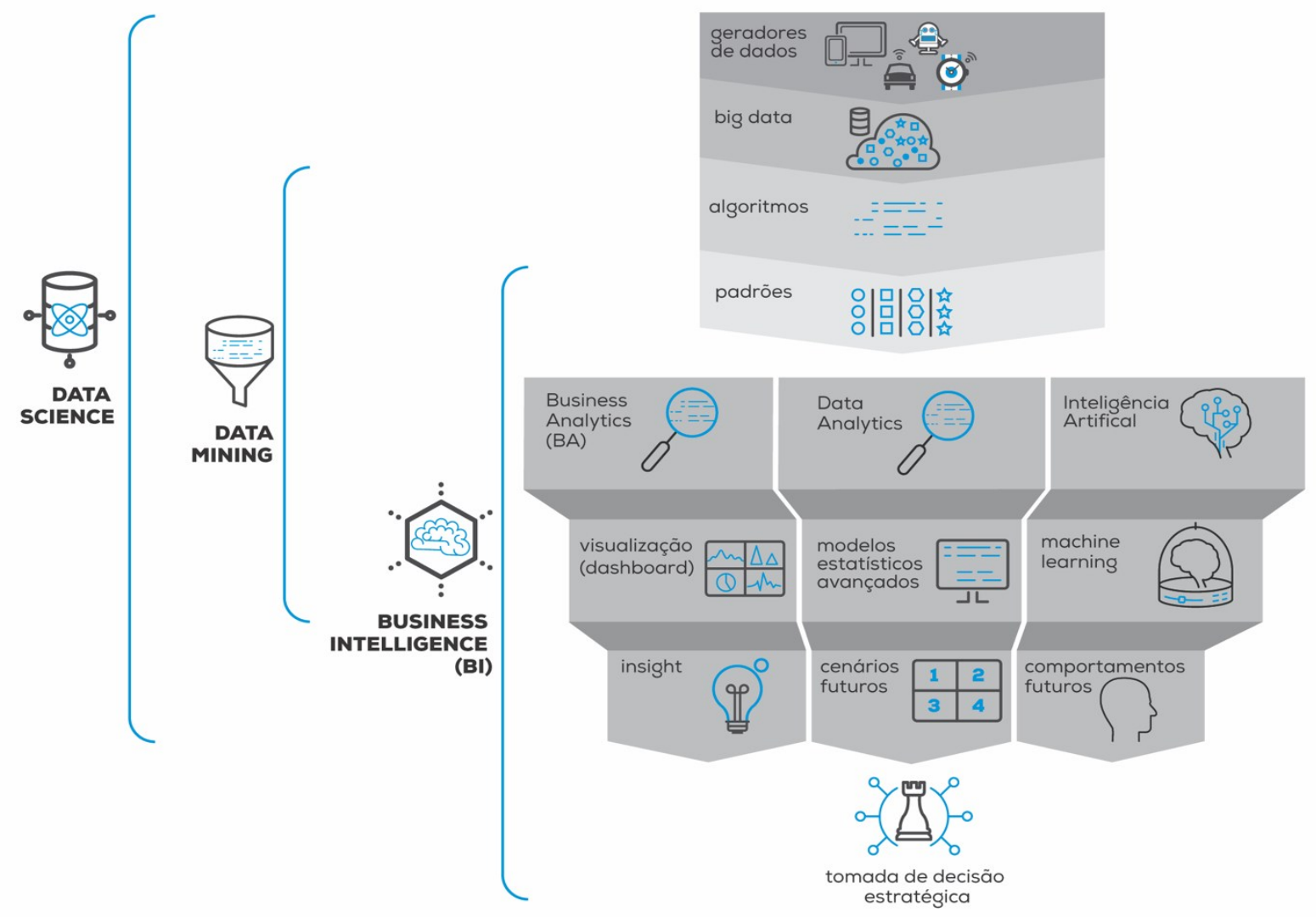

FIGURA 1 - Tecnologias relacionadas ao Big Data \& Design. Fonte: Os autores.

O Data Science é a ciência orientada a dados que engloba preparação, limpeza e análise de dados, como a mineração de dados (Data Mining). Enquanto, o Business Intelligence (BI) é definido como um conjunto de processos e ferramentas de coleta, análise e de dados utilizados para uma tomada de decisão estratégica (DAVENPORT; SPANYI, 2016).

Algumas das tecnologias atreladas ao Data Science, Data Mining e BI são: (1) Business Analytics (BA) que trata dos aspectos analíticos necessários para se atingir o BI (DAVENPORT, 2020); (2) Data Analytics que envolve a aplicação de processos modelos estatísticos avançados para projetar cenários futuros; (3) Tecnologias de Inteligência artificial (Artificial Intelligence - AI) e Aprendizado de Máquina (Machine Learning - ML) que podem ser utilizadas para mapear comportamentos futuros por meio da compreensão e imitação da inteligência humana. Para que haja dados para esses processos, usuários e outras tecnologias precisam gerá-los sendo a IoT (Internet of the Things) uma das principais delas. Conforme Oliana, Constantinides e Vries (2018), a IoT é uma rede composta por diferentes dispositivos inteligentes, equipados com sensores e softwares que coletam e trocam dados.

Neste cenário, Costa e Pelegrini (2019) apontam que todas essas novas tecnologias estão impactando diretamente o Design e o desenvolvimento de produtos, serviços ou sistemas. 
Sendo assim, a abordagem de DDD apresenta-se pertinente para a inovação no PDP (figura 2), visto que é associada a uma tomada de decisão fundamentada em dados digitais quantitativos.

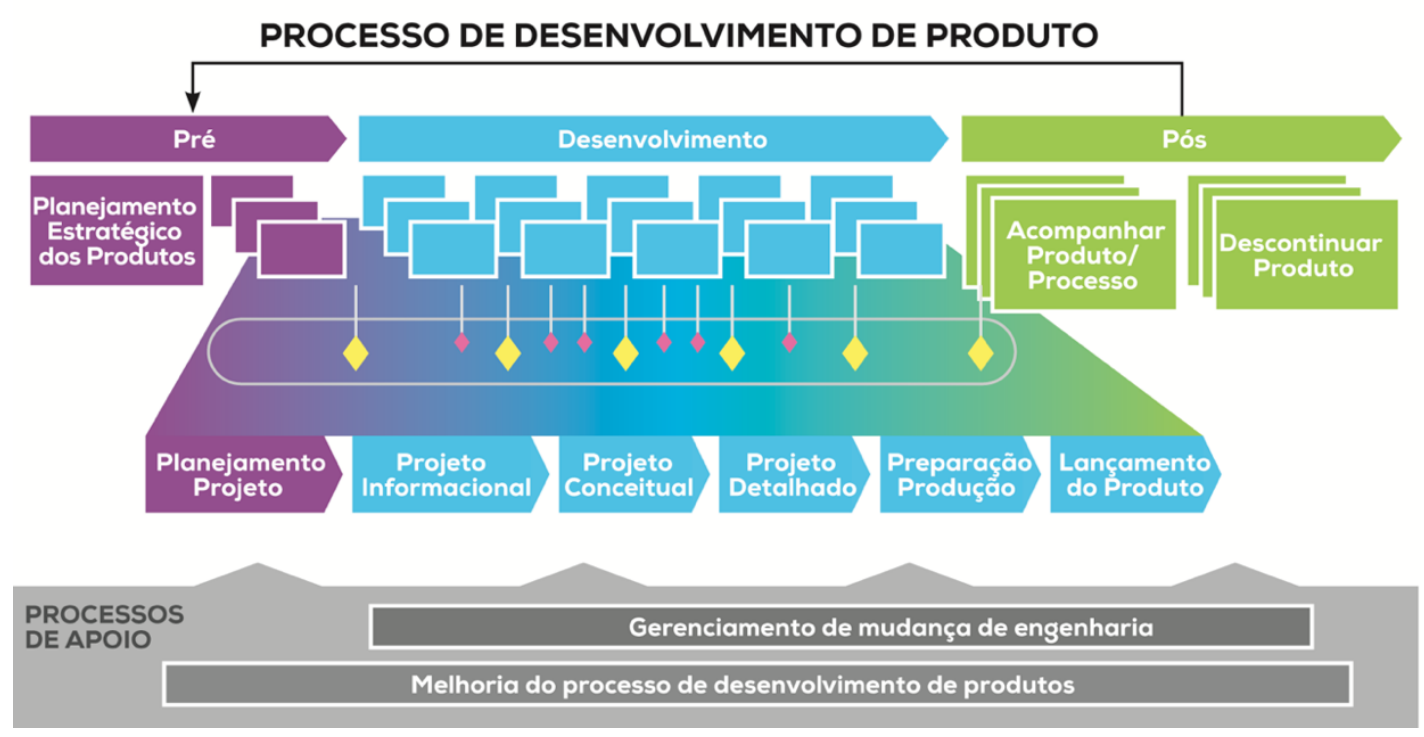

FIGURA 2 - Modelo de Referência do PDP. Fonte: Baseado em Rozenfeld et al. (2006).

Conforme Tan e Zhan (2017), alguns setores já estão se formalizando, unificando e inovando o PDP, através do uso das potencialidades oferecidas pelas novas TIC. Provost e Fawcet (2016) também apontam que desta maneira pode haver um melhor gerenciamento de produtos e a tomada de decisão é mais aprimorada, elevando o desempenho dos negócios.

\section{METODOLOGIA}

A presente pesquisa possui abordagem quantitativa, caráter exploratório, análise crítica da literatura e natureza descritiva. O método adotado para levantamento de dados foi uma Revisão Bibliográfica Assistemática (RBA), seguida de uma Revisão Bibliográfica Sistemática (RBS).

A RBA permitiu estruturar a fundamentação teórica, identificar os principais temas e as possíveis palavras-chave para a RBS. A RBS foi conduzida através de um roteiro (CONFORTO et al., 2011) composto por 3 fases (Entrada, Processamento e Saída). Na entrada, foram definidos o problema, os objetivos e critérios de inclusão: artigos revisados por pares, publicados de 2015 a 2020, em português ou inglês. Além disso, foram delineadas as fontes primárias em bases com rigor de indexação: Periódicos Capes, Science Direct e Scopus. Com relação aos termos (strings), foram definidos dois grupos (que foram cruzados no processo de busca), sendo o primeiro composto por termos relacionados à ciência de dados: big data, data mining, business intelligence, data-driven design e business analytics. Enquanto o segundo 
relaciona-se ao PDP: new product development, product innovation processes, NDP, PDP e design process.

\section{RESULTADOS E DISCUSSÃO}

A partir das buscas foram identificados 3.253 artigos. Na fase de processamento, após a exclusão de artigos de outros campos, 1.231 publicações passaram para o Filtro 1. Destes foram lidos títulos, resumos e palavras-chaves, passando 113 artigos para o Filtro 2, dos quais foram lidas introduções e conclusões, resultando 39 artigos. Na fase de saída, com Filtro 3, os artigos foram lidos integralmente, resultando em 16 trabalhos.

Os autores, o ano de publicação e as principais contribuições dos artigos lidos constam no gráfico 1. A pontuação refere-se a quanto o trabalho está próximo desta pesquisa, sendo: "1" próximo e " 2 " muito próximo.

\begin{tabular}{|r|l|l|}
\multicolumn{1}{|l|}{ artigo, ano } & principal contribuição & \\
\hline BERTONI, 2020 & Investiga definições, usos e aplic ação do DDD na conceitualização do PDP & \\
\hline DE SILVA et al., 2019 & Uso da Realidade Virtual e Aumentada para melhoria no NPD & \\
\hline HAJLI et al., 2019 & Entendimento rápido (big data) sobre o cliente para o NPD & \\
\hline TIMOSHENKO e HAUSER, 2019 & Uso de ML para análise qualitativa e identificação das necessidades do usuário & \\
\hline GRÜTZMANN, et al., 2019 & Uso de tecnologias de internet para inovação de produtos em empresas brasileiras & \\
\hline DARCO, et al., 2019 & Framework sobre o uso do Big Data e IA para explorar e gerenciar a jornada do usuário & \\
\hline LI, et al., 2019 & Modelo integrado para PDP de novos smart products, usando data-driven & \\
\hline ZABOTTO et al., 2019 & Modelo teórico de Kansei para a criação de moodboards automáticos com base em Big Data & \\
\hline ZHAN et al., 2018 & Uso de Big Data para conectar-se e interagir com clientes no NPD & \\
\hline OLIANA et al., 2018 & Uso de dados de loT no processo de desenvolvimento de NPD & \\
\hline CHRISTENSEN et al., 2018 & Modelo de extração de ideias com base em dados de redes sociais & \\
\hline RATHORE et al., 2018 & Análisa conversacionalmente dados de mídia social sobre as percepções dos usuários & \\
\hline BERTONI, 2018 & Discute a função e os desafios da integração do DDD ao processo de inovação de NPD & \\
\hline TAN e ZHAN, 2017 & Big Data aplicado no NPD para melhorar a adoção de produtos e reduzir custos & \\
\hline WILBERG et al., 2017 & Framework para apoiar o desenvolvimento de estratégia de dados para PDP & \\
\hline RATHORE, et al., 2016 & Conceitua e discute os possiveis insights advindos da análise de dados de mídia social & \\
\hline & & \\
\hline
\end{tabular}

GRÁFICO 1 - RBS: Resultados. Fonte: Os autores.

Vê-se no gráfico que 50\% dos artigos foram publicados entre 2019 e 2020, revelando que a intersecção dos temas tem sido recente e um assunto em ascensão. Ademais muitos dos estudos utilizam o Big Data para compreender o comportamento do usuário para inovação no PDP. 


\subsection{Fontes de Big Data}

Ao tratar dos tipos e fontes de Big Data, para Timoshenko e Hauser (2019) a complexidade desses gera dificuldade no gerenciamento do processo de PDP. Ao mesmo tempo, segundo D'arco et al. (2019) e Wilberg et al. (2017), a capacidade de desenvolver produtos conectados entre si e com a nuvem, tem criado fontes ricas em dados. $O$ gráfico 2 ilustra as principais fontes de dados resultantes da RBS.

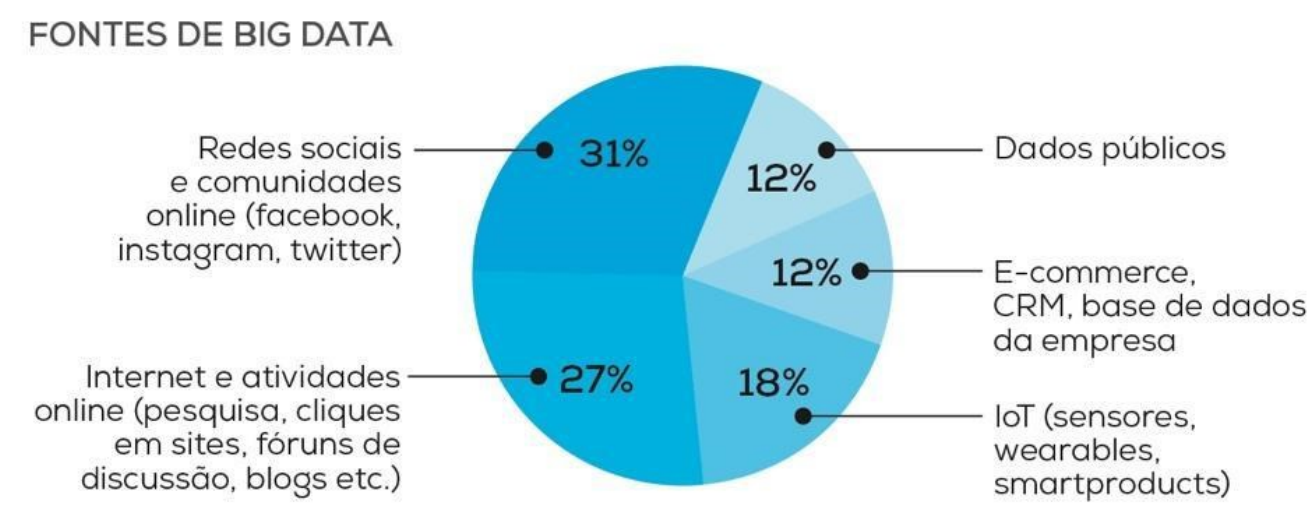

GRÁFICO 2 - RBS: principais fontes de dados utilizadas no PDP. Fonte: Os autores.

Conforme o gráfico, alguns dados aplicados no PDP advêm da interação do usuário com a internet. As redes sociais e comunidades online são as fontes mais utilizadas, representando $31 \%$ das atividades citadas nos artigos. Outras interações online, como pesquisa em sites de busca, cliques em sites, fóruns de discussão e blogs respondem por $27 \%$ das fontes, apresentando dados de natureza comportamental, psicográfica e demográfica (D'ARCO et al., 2019). Nota-se que o uso de IoT tem sido também utilizado (18\%), os dados derivados do uso do produto, em tempo real, também compõem este. Já dados de e-commerce, CRM e base de dados internos da empresa apresentam dados de natureza transacional, histórica e demográfica (D’ARCO et al., 2019), e são responsáveis por apenas 12\% das fontes. Vê-se que as fontes de dados abertos ou públicos também são pouco utilizadas (12\%).

\subsection{Tecnologias para captação de Big Data}

Observa-se no gráfico 3 a predominância do uso do Data Mining de texto e análise de conteúdo e de sentimentos (56\%). Em segundo lugar ficou o ML, com 32\% de uso nos artigos. Com relação a análise desses dados há predominância de análises de fontes de redes sociais, principalmente em text mining (BERTONI, 2020; CHRISTENSEN et al., 2018; 
GRÜTZMANN; ZAMBALDE; BERMEJO, 2019; HAJLI et al., 2019; RATHORE; ILAVARASAN; DWIVEDI, 2016), isso pode justificar-se pela facilidade de acesso a esses dados. As filtragens léxicas e ML também permitem realizar uma análise de sentimentos via Big Data do UGC (User Generated Content - conteúdo gerado pelo usuário). Sendo este um dos métodos para se identificar as emoções e obter insights do usuário (D'ARCO et al., 2019).

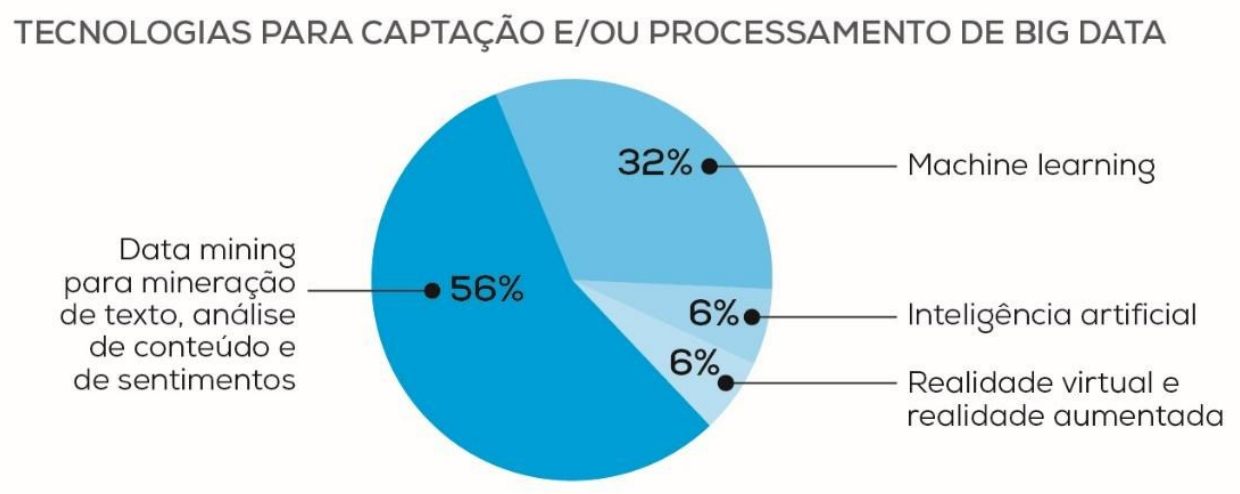

GRÁFICO 3 - RBS: principais tecnologias para coleta e/ou processamento de dados. Fonte: Os autores.

A AI em conjunto com as realidades virtual (VR) e aumentada (AR), representam apenas 12\% das tecnologias utilizadas, acredita-se que seja pelo fato destas tecnologias serem mais específicas.

\subsection{Big Data e as Macrofases do PDP}

Grützmann, Zambalde e Bermejo (2019) e Zahay, Griffin e Fredericks (2004) apontam que as necessidades de informações são irregulares no processo de PDP. Sendo que as macrofases mais exigentes são o pré-desenvolvimento e o desenvolvimento, o que pode justificar o elevado número de artigos que tratam dos dados nestas fases, 69\% conforme o gráfico 4 .

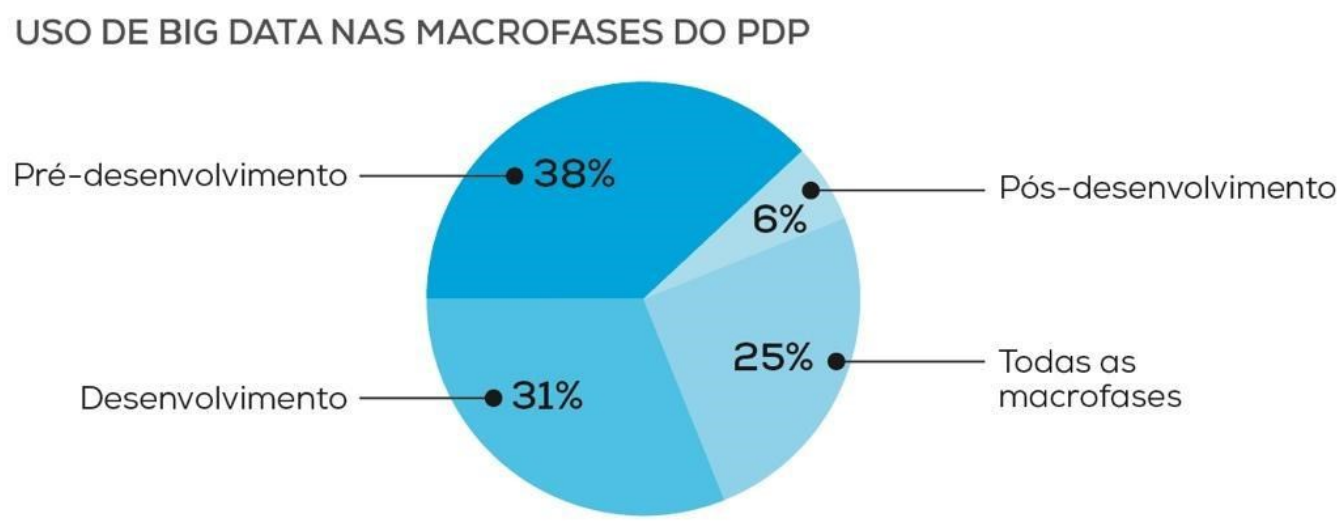

GRÁFICO 4 - RBS: uso de Big Data nas macrofases do PDP. Fonte: Os autores. 
Na sequência, durante as etapas de teste e a validação, o número de tipos de informações que podem ser úteis no gerenciamento do projeto aumenta novamente, porque reflete um conjunto mais amplo de incertezas. Posteriormente, verifica-se no pós-desenvolvimento uma baixa de utilização de Big Data (6\%) onde as maiores aplicações são em ações de marketing direcionadas à venda e manutenção (D'ARCO et al., 2019).

\subsection{Big Data \& pré-desenvolvimento do PDP}

No pré-desenvolvimento do PDP, o primeiro passo para utilização de Big Data deve descrever uma estratégia integrada de dados que dê suporte à decisão (WILBERG et al., 2017). Bertoni (2018) e Li, Roy e Saltz (2019) frisam que esta fase deve-se permitir lidar com situações em que dados disponíveis são escassos, e a informação pode estar imatura, típico de um contexto de Fuzzy Front End. Ademais, as empresas precisam garantir que todos os membros da equipe tenham uma linguagem comum, obtendo um mesmo significado dos dados e que sejam desenvolvidos sistemas de gerenciamento de informações para garantir que se atendam às necessidades em todas as macrofases (LI et al., 2019; ZAHAY; GRIFFIN; FREDERICKS, 2004).

Nesta macrofase, o Big Data permite a integração de clientes e os transforma em fontes para apoiar as empresas na geração e avaliação de ideias (D'ARCO et al., 2019; ZABOTTO et al., 2019; ZHAN et al., 2018). A maioria das contribuições dizem respeito à identificação das necessidades dos clientes (BERTONI, 2020). Wilberg et al. (2017) apontam que dados relacionados ao uso de produtos também podem trazer novas estratégias ao identificar problemas.

Timoshenko e Hauser (2019) sugerem o uso de ML para a compreensão da experiência do cliente, ao contrário de métodos tradicionais que são mais caros, demorados e recaem em julgamento humano. O conteúdo gerado pelo usuário, como análises online, mídias sociais e blogs, fornece dados textuais significantes e abrangentes na identificação de necessidades (D'ARCO et al., 2019).

Conforme Christensen et al. (2019) é possível extrair insights para gerar inovações com base em textos de usuários de redes sociais usando ML e Data Mining. Os autores argumentam que o método de marcadores linguísticos é aplicável em diferentes áreas tecnológicas e categorias de produtos, porque a maioria das pessoas usa um conjunto específico de palavras e expressões ao se comunicarem. 
Vê-se que as mídias sociais criam muitas oportunidades para a identificação e engajamento do público. Assim, as organizações podem comparar aspectos relativos ao produto com as percepções individuais (RATHORE et al., 2018). Sendo que o perfil do consumidor e de personas podem ser construídos com base nessas ações online, sejam voluntárias ou não (D'ARCO et al., 2019).

\subsection{Big Data \& desenvolvimento do PDP}

O Big Data suporta o desenvolvimento através da coleta de dados para geração de insights. Nesta macrofase são necessárias informações claras e objetivas. Porém, uma dificuldade encontra-se nas diferenças de linguagens utilizadas no processo, devido à variedade de profissionais envolvidos, sendo de área técnica, de marketing, de ciência de dados (TAN; ZHAN, 2017), de Design, dentre outras.

Ao tratar do uso de dados com base em VR e AR, este agiliza o processo, fazendo com que produtos virtuais possam ser utilizados para o processo de tomada de decisão antecipada (SILVA et al., 2019). O Big Data também pode acelerar o lançamento de produtos e determinar os pontos fracos no início do desenvolvimento (TAN; ZHAN, 2017).

Para a etapa de geração de alternativas, o suporte à decisão de um projeto deve permitir que a equipe selecione os conceitos mais assertivamente. Assim, iterações via Big Data podem ser executadas em tempo real, e um primeiro lote de modelos podem ser disponibilizados rapidamente (BERTONI, 2018).

Conforme Bertoni (2018) e Wilberg et al. (2017), o principal uso dos dados no desenvolvimento serve para aperfeiçoar a manutenção do produto captando dados de uso. Esses podem ser analisados para agrupar clientes, monitorar a condição do produto, dificuldades e erros de uso.

Para a atividade de criação, as estratégias de cocriação e colaboração também podem ser alavancadas (RATHORE; ILAVARASAN; DWIVEDI, 2016; ZHAN et al., 2018) na medida em que esta abordagem permite que os clientes "projetem" produtos que atendam às necessidades particulares. A vantagem de usar Big Data envolvendo o cliente, ao contrário de pesquisas de mercado convencionais, é que os clientes não são apenas questionados sobre suas necessidades, eles podem exibir sua criatividade. Isso pode ser atingido com simulações ou aquisições de informações de diferentes fontes relativas a um produto, individualizando-se os protótipos virtuais (GRÜTZMANN; ZAMBALDE; BERMEJO, 2019). Zhan et al. (2018) também indica que para testes e lançamento do produto, o Big Data permite que as empresas 
transfiram indivíduos de diferentes fontes (comunidades, sites e plataformas baseados na Web) para o papel de possíveis clientes.

\subsection{Big Data \& pós-desenvolvimento}

No pós-desenvolvimento é importante acompanhar a performance do produto no mercado. Tan e Zhan (2017) citam que empresas que se comunicam com seus clientes por meio de Big Data, reduzem custos e tempo.

No contexto brasileiro, Grützmann, Zambalde e Bermejo (2019) mostram que empresas fazem investimentos em várias frentes de tecnologia nesta macrofase. Os blogs, fóruns de discussão e mídias sociais, em geral, reúnem as informações para o pós-lançamento e manutenção.

Ao tratar da jornada do usuário, D'arco et al. (2019) apresentam um framework sobre o uso do Big Data e IA. Os autores demonstram como a IA pode suportar sistemas de tomada de decisão e apontam o uso de Big Data em atividades do pós-desenvolvimento, como construir estratégias de venda, de preço e promoções, também para aquisição de clientes e previsão de demanda. Como fontes de dados para estas atividades são considerados principalmente dados de ecommerce e de CRM da empresa, mas também dados de redes sociais e atividades online.

\subsection{Atividades das macrofases do PDP \& origem dos dados}

O quadro 1 representa as macrofases do PDP e suas atividades, onde é levado em conta, principalmente, a inteligência que o Big Data pode prover e o tipo de dados que apoia cada atividade. 


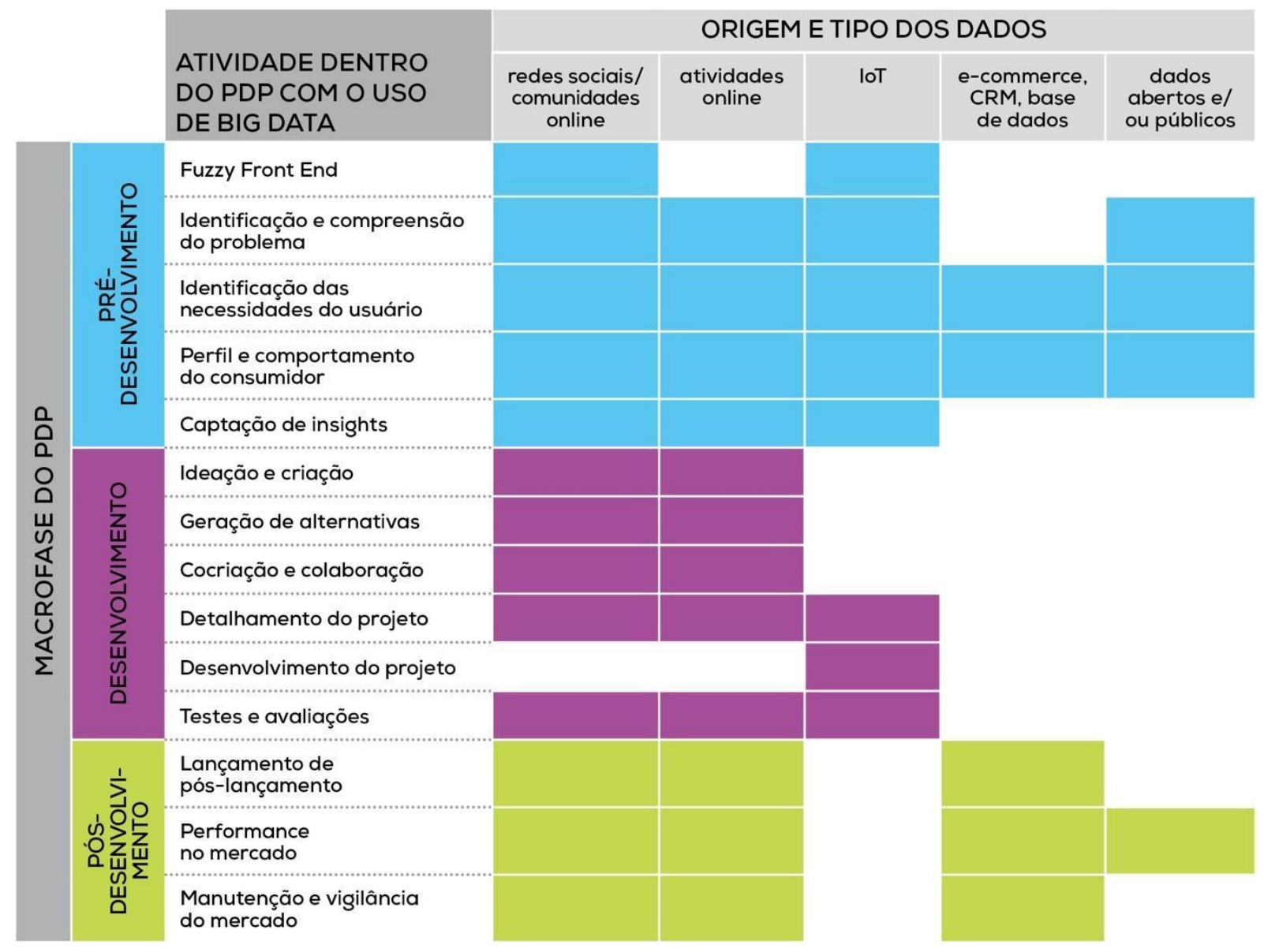

QUADRO 1 - Atividades nas macrofases e fontes de dados. Fonte: Os autores.

Vê-se que a macrofase do pré-desenvolvimento é a que utiliza a maior variedade de dados para suas atividades. Já o desenvolvimento utiliza dados mais relacionados a interações nas redes sociais e de todas as atividades online. Por fim, o pós-desenvolvimento utiliza mais dados de transações comerciais como de e-commerce, CRM e dados internos.

Quanto às atividades levantadas (as que mais foram citadas nos resultados da RBS), observouse que a atividade de formulação estratégica foi pouco abordada. A etapa de descontinuidade do produto, pensando na circularidade e sustentabilidade, também não foi abordada.

\section{CONCLUSÃO}

$\mathrm{O}$ artigo apresentou os resultados de uma revisão sistemática da literatura investigando a aplicação do DDD no PDP. As contribuições científicas relevantes para a pergunta de pesquisa foram categorizadas com base no uso de Big Data nas diferentes macrofases do PDP. A análise focou nas tecnologias, origens, tipo e processamento de dados dentro das macrofases e suas contribuições para o PDP. 
Mostrou-se uma predominância do uso de técnicas de mineração de texto provenientes das mídias sociais, além de análises online para todas as macrofases do PDP. No prédesenvolvimento e desenvolvimento são obtidos dados com intuito de identificar problemas, perfil e necessidades dos clientes e usuários. No desenvolvimento, esta mesma origem de dados alimenta a ideação, criação, geração de alternativas, detalhamento do projeto, testes e avaliações. Já no pós-desenvolvimento, apesar de ter sido observado um menor uso do DDD, esses são usados para apoiar atividades como lançamento, acompanhamento da performance de produto e manutenção. Os dados utilizados nesta macrofase são, além de também provenientes de redes sociais, derivados de sistemas de e-commerce, CRM e banco de dados da organização. Apesar dessa segmentação quanto à origem do Big Data, para as empresas que pretendem extrair conhecimento dos grandes dados para apoiar o processo de inovação, sugere-se a utilização de múltiplas origens e tecnologias em todas as macrofases. Os resultados evidenciam que a inteligência com base em dados deve estar alinhada estrategicamente na organização, fazendo com que os diversos profissionais envolvidos sigam a mesma linguagem e planejamento.

Quanto às contribuições, destaca-se a possibilidade de acelerar o processo determinando os pontos fracos antecipadamente e a utilização de dados de uso em tempo real para aperfeiçoar o desenvolvimento. A facilitação da colaboração, em tempo real, dos clientes por meio de plataformas de cocriação, também se destaca. Apesar de não serem o foco deste estudo, e não terem sido suficientemente abordados pelos autores, aspectos legais impõe desafios e barreiras relacionados a coleta, armazenagem e utilização de Big Data. No Brasil, de forma a garantir que esses processos ocorram de maneira ética, transparente e com segurança, em 2020 entrou em vigor a LGPD (Lei Geral de Proteção de Dados). Ademais, outras lacunas encontradas referem-se à aplicação de dados em etapas estratégicas e para sustentabilidade.

Por fim, os resultados parecem não estar alinhados com a teoria que descreve as abordagens DDD como uma resposta à oportunidade concedida pelo aumento da acessibilidade da ciência de dados. Nota-se que ainda há muito a se evoluir no que tange a estruturação do PDP para planejar sistematicamente a implementação do DDD. Desta maneira, o artigo fornece um ponto de partida para investigações mais profundas, levando adiante o estado da arte do DDD aplicado ao PDP para a inovação. 


\section{REFERÊNCIAS}

ALÃO, R. Sobre a complexidade dos problemas contemporâneos de design. DAT Journal, v. 5, n. 4, p. 24-40, 2020.

BERTONI, A. Role and challenges of data-driven design in the product innovation process. IFACPapersOnLine, v. 51, n. 11, p. 1107-1112, 2018.

BERTONI, A. Data-driven design in concept development: systematic review and missed opportunities. In: DESIGN SOCIETY: design conference, 2020. Proceedings [...] Cambridge: Cambridge University Press, v. 1, p. 101-110. DOI: https://doi.org/10.1017/dsd.2020.4

CHRISTENSEN, K.; SCHOLDERER, J.; HERSLETH, S. A.; NÆS, T.; KVAAL, K.; MOLLESTAD, T.; RISVIK, E. How good are ideas identified by an automatic idea detection system?. Creativity and Innovation Management, v. 27, n. 1, p. 23-31, 2018.

CONFORTO, E. C.; AMARAL, D.C.; SILVA, S.L. Roteiro para revisão bibliográfica sistemática: aplicação no desenvolvimento de produtos e gerenciamento de projetos. In: CONGRESSO BRASILEIRO DE GESTÃO DE DESENVOLVIMENTO DE PRODUTOS, 8., 2011, Porto Alegre. Anais [...] Porto Alegre: UFRGS, 2011. p. 112.

COSTA, C.; PELEGRINI, A. Modelo para estabelecer competências para o futuro do design orientado pelas tecnologias emergentes. Estudos em Design, v. 27, n. 3, p.180-198, 2019.

D'ARCO, M. D; PRESTI, L. L.; MARINO, V.; RESCINITI, R. Embracing AI and Big Data in customer journey mapping: from literature review to a theoretical framework. Innovative Marketing, v. 15, n. 4, p.102-115, 2019.

DAVENPORT, T.; SPANYI, A. Improve new product development with predictive analytics. 2016. Disponível em: http://ide.mit.edu/news-blog/blog/improve-new-product-development-predictive-analytics. Acesso em: 21 abr. 2020.

DEVI, G. N. R. Emerging trends in big data analytics - a study. In: KIMAR, A.; MOZAR, S. (Ed.). ICCCE: proceedings of the international conference on communications and cyber physical engineering. Singapore: Springer, 2019. p. 563-570. (Lecture Notes in Electrical Engineering, v.500)

FRISK, J. E.; BANNISTER, F. Improving the use of analytics and big data by changing the decision-making culture: a design approach. Management Decision, v. 55, e.10, p. 2074-2088, 2017.

GRÜTZMANN, A.; ZAMBALDE, A. L.; BERMEJO, P. H. S. Innovation, new product development and internet technologies: study in Brazilian companies. Gestão \& Produção, v. 26, n. 1, 2019. DOI: https://doi.org/10.1590/0104-530X1451-19

HAJLI, N.; TAJVIDI, M.; GBADAMOSI, A.; NADEEM, W. Understanding market agility for new product success with big data analytics. Industrial Marketing Management, v. 86, p. 135-143, 2019.

LI, Y.; ROY, U.; SALTZ, J. S. Towards an integrated process model for new product development with datadriven features (NPD 3). Research in Engineering Design, v. 30, n. 2, p. 271-289, 2019.

OLIANA, R.; CONSTANTINIDES, E.; VRIES, S. The Internet of things: the next big thing for new product development? Journal of Marketing Trends, v. 5, n.1. p. 159-176, 2018.

PRIOR, V. Glossary of terms used in competitive intelligence and knowledge management. Virginia: SCIPStrategic and Competitive Intelligence Professionals, 2010.

PROVOST, F.; FAWCETT, T. Data science para negócios. Rio de Janeiro: Alta Books, 2016.

RATHORE, A. K.; DAS, S.; ILAVARASAN, P. V. Social media data inputs in product design: case of a smartphone. Global Journal of Flexible Systems Management, v. 19, n. 3, p. 255-272, 2018.

RATHORE, A. K.; ILAVARASAN, P. V.; DWIVEDI, Y. K. Social media content and product co-creation: an emerging paradigm. Journal of Enterprise Information Management, 2016.

ROZENFELD, H.; AMARAL, D.C.; ALLIPRANDINI, D.H.; FORCELLINI, F.; TOLEDO, J.C.; SCALICE, R. SILVA, S.L. Gestão de projetos em desenvolvimento de produtos: uma referência para a melhoria do processo. São Paulo: Saraiva, 2006. 
SILVA, R. K. J.; RUPASINGHE, T. D.; APEAGYEI, P. A Collaborative apparel new product development process model using virtual reality and augmented reality technologies as enablers. International Journal of Fashion Design, Technology and Education, v. 12, n. 1, p. 1-11, 2019.

SPIEGEL, R. Home big data and cloud computing have moved into design. Design News. v. 71, e. 10, p. 38-39, 2016.

TAN, K. H.; ZHAN, Y. Improving new product development using big data: a case study of an electronics company. R\&D Management, v. 47, n. 4, p. 570-582, 2017.

TIMOSHENKO, A.; HAUSER, J. R. Identifying customer needs from user-generated content. Marketing Science, v. 38, n. 1, p. 1-20, 2019.

WILBERG, J.; TRIEP, I.; HOLLAUER, C.; OMER, M. Big Data in product development: need for a data strategy. In: INTERNATIONAL CONFERENCE ON MANAGEMENT OF ENGINEERING AND TECHNOLOGY, 2017, Portland. Proceedings [...] Piscataway: IEEE, 2017. DOI: 10.23919/PICMET.2017.8125460

ZABOTTO, C. N.; AMARAL, D. C.; HORNOS, C. J. M.; BENZE, B. G. Automatic digital mood boards to connect users and designers with kansei engineering. International Journal of Industrial Ergonomics, v. 74, Nov. 2019. DOI: 10.1016/j.ergon.2019.102829.

ZAHAY, D.; GRIFFIN, A.; FREDERICKS, E. Sources, uses, and forms of data in the new product development. Industrial Marketing, v. 33, n. 7, p. 657-666, 2004.

ZHAN, Y.; TAN, K. H.; LI, Y.; TSE, Y. K. Unlocking the power of big data in new product development. Annals of Operations Research, v. 270, n. 1, p. 577-595, 2018. 